\title{
Isolation and Characterization of Volatile Organic Compounds-Degrading Bacillus Strains from Loess
}

\author{
Soo Yeon Lee, Hye Yun Oh, Ok Bin Kim
}

Department of Life Sciences, Ewha Womans University, Seoul, Korea.

Email: kimokbin@ewha.ac.kr

Received February $4^{\text {th }}, 2013$; revised March $9^{\text {th }}, 2013$; accepted April $11^{\text {th }}, 2013$

Copyright (C) 2013 Soo Yeon Lee et al. This is an open access article distributed under the Creative Commons Attribution License, which permits unrestricted use, distribution, and reproduction in any medium, provided the original work is properly cited.

\begin{abstract}
Volatile organic compounds (VOCs) are harmful to human health and the environment. Recently, loess (Hwangtoh) was used as an eco-friendly interior paint formulation in Korea. It is used even more commonly as a filter carrier to remove VOCs. In this study, we isolated Bacillus strains from a loess filter. The strains that were tolerant to VOCs were labeled according to the series VOC01 to VOC35. Four strains-VOC03, VOC11, VOC18, and VOC30 - were investigated for their ability to degrade cyclohexane and toluene. Strain VOC18 best degraded both VOCs, whereas VOC03 demonstrated no ability to degrade VOCs. In keeping with this, VOC18 grew best on cyclohexane or toluene as the sole carbon source. The strains were identified by their physiochemical and phylogenetic characteristics. Strain VOC18 was determined as a strain of Bacillus cereus; VOC11 and VOC30 were determined as differentiated strains of B. thuringiensis. Strain VOC03, which demonstrated high tolerance but no ability to degrade VOCs, was identified as a strain of B. megaterium.
\end{abstract}

Keywords: Loess Filter; Volatile Organic Compounds (VOCs); Bacillus; Cyclohexane; Toluene

\section{Introduction}

Volatile organic compounds (VOCs) are organic compounds that evaporate easily into gas due to their high vapor pressure resulting from a low boiling point at ordinary room temperature under normal atmospheric pressure. VOCs include hydrocarbons, halogenated hydrocarbons, and nitrogen- and sulfur-containing hydrocarbons. Many VOCs are dangerous to human health, as they are either carcinogenic or otherwise hazardous. VOCs are also harmful to the environment. They can cause photochemical smog in the atmosphere, destruct the stratospheric ozone layer, and ultimately play a role in global warming [1-4]. Since Drake's discovery of an oil field in 1859 , petroleum resources have been used as the main source of power for the entire industry and have enabled the current industrial development. Starting with Rockefeller's oil companies, the oil industry has been refining raw oil to produce not only fuels but also various petroleum-based chemicals, and the types and amounts of these products have been increasing. Through the process of industrialization, the production and consumption of petroleum-based products has increased, and accordingly, the emissions of various types of VOCs into our surroun- dings have been increasing significantly. Enormous amounts of carbon, 110 - 150 teragram, are emitted annually in the form of VOCs as a result of these human activities $[5,6]$. These VOCs are emitted from large-scale industrial facilities such as petroleum refineries, organic synthesis facilities, and manufacturing plants. In addition, they are also emitted from various machines used on a daily basis, such as dry cleaning equipment and automobiles. The concentration levels of these anthropogenic VOCs in the atmosphere are regulated by the law, particularly the concentration level of indoor VOCs, which is found to be the highest. After the report of the World Commission on Environment and Development (WCED) was published, global environmental regulations were fortified in an effort to ensure sustainable environmental protection and economic development. Therefore, the laws and policies pertaining to environmental regulations such as $\mathrm{REACH}$, WEEE, and RoHS have been continually updated and developed. In addition, there has been a trend toward replacing the existing chemical purifying processes with more environmentally friendly biological processes $[7,8]$. In particular, the bioremediation of VOCs using a biofilter with microorganisms has a lower cost and is more environmentally friendly than physical and chemical processes. 
Before industrialization, VOCs that occurred naturally were recycled within the corresponding environment. Soil microorganisms play a major role in the degradation of VOCs in the environment. It has been known to study well on the degradation of VOCs, including cyclohexane, by metabolically versatile Pseudomonas as well as by other gram-negative strains such as Xanthobacter or Brachymonas has been well studied [9-12]. The degradation of VOCs by gram-positive bacteria, which are generally less tolerant to VOCs than gram-negative bacteria [13], has been reported for benzene utilizing Rhodococcus [14], cyclohexane-degrading Rhodococcus [15], and recently, diesel-degrading Bacillus [16]. In the recent years, in Korea, there have been an increasing number of cases in which loess has been used as an eco-friendly interior paint formulation or as a filter material for the purification of indoor air pollutants. Microorganisms that inhabit loess can be used to remove VOCs or to convert them to nontoxic chemicals. In the present study, we isolated soil bacterial strains from loess and investigated whether the strains play a role in the degradation of VOCs. Four Bacillus strains that grew well with VOCs were selected. The phenotypic character of the four strains was studied along with their morphological and physiochemical properties, and the phylogenetic position of the strains was also characterized by a comparison of $16 \mathrm{~S}$ rDNA sequences.

\section{Materials and Methods}

\subsection{Isolation of VOC-Tolerant Strains and Growth Conditions}

The loess (Hwangtoh) was collected from a biofilter (IL WOO TECH Co., Ltd.) that was exposed to gasoline (at a local gas station), used as a VOC, for $50 \mathrm{~h}$ in a sealed container. The samples were extracted using saline solution $(0.85 \% \mathrm{NaCl})$, and the extracts were serial diluted. The diluted samples were distributed onto an LB (Lauria-Bertani)- or TSB-agar plate (Difco) and incubated at temperatures $30^{\circ} \mathrm{C}$ and $37^{\circ} \mathrm{C}$. The microbes were sorted based on morphology, color, and surface type of the colonies, and they were cultivated until a pure culture was obtained. To select the VOC-tolerant strains, the strains were grown in an LB- or BH (Bushnell-Hass)-agar plate covered with $150 \mathrm{ml}$ of toluene (99.9\%, Sigma-Aldrich), cyclohexane (99.7\%, Sigma-Aldrich), benzene (99.9\%, Sigma-Aldirich), or gasoline (local store) [17] at $30^{\circ} \mathrm{C}$.

For the growth experiment, subcultures of VOC strains were grown in an LB medium containing $1 \%(\mathrm{v} / \mathrm{v})$ of the VOC (toluene, cyclohexane, benzene, or gasoline) at $30^{\circ} \mathrm{C}$, placed overnight on a shaking incubator (200 rpm). The main cultures were inoculated with $1 \%$ of subculture $(\mathrm{v} / \mathrm{v})$ in a modified BH medium (Bushnell-Hass agar) [17] containing $1 \%(\mathrm{v} / \mathrm{v})$ of VOCs (toluene, cyclohexane, ben- zene, or gasoline), and incubated at $30^{\circ} \mathrm{C}$ with shaking at $200 \mathrm{rpm}$ for 7 days. The growth of 4 VOC strains on the VOCs was indicated in terms of $\Delta \mathrm{OD}_{600}$ and $\mathrm{Y}_{\text {sub }}$ (grams of cell dry weight per mole). The substrate molar yield $\left(\mathrm{Y}_{\text {sub }}\right)$ was expressed as the cell dry weight per mole of the carbon source.

\subsection{Degradation of Toluene and Cyclohexane by VOC Strains}

For the VOC degradation experiment, the strains were grown aerobically in a $\mathrm{BH}$ liquid medium with $1 \%(\mathrm{v} / \mathrm{v})$ of toluene or cyclohexane at $30^{\circ} \mathrm{C}$ on a shaking incubator (180 rpm) up to $\mathrm{OD}_{600}>1$. After removal of the old medium by centrifugation, the cells of the strains were resuspended in a fresh $\mathrm{BH}$ medium to an $\mathrm{OD}_{600}$ of 0.5. The $50 \mathrm{ml}$ resuspension was transferred with toluene or cyclohexane to $500 \mathrm{ml}$ serum bottles. The bottles were plugged with rubber stoppers and completely sealed with aluminum caps. While the cells were cultivated on the shaking incubator at $30^{\circ} \mathrm{C}$, the gas in the headspace of the serum bottles was sampled using a gas-tight syringe (Hamilton Co., NV, USA). Toluene or cyclohexane in the gas samples was analyzed by Gas Chromatography (GC6850N, Agilent Technologies Inc.) with a wax column (30 $\mathrm{m} \times$ $0.32 \mathrm{~mm} \times 0.25 \mu \mathrm{m}$, Supelco, USA) and an FID detector. The oven temperature was maintained at $100^{\circ} \mathrm{C}$ for $5 \mathrm{~min}$ and then increased to $320^{\circ} \mathrm{C}$ at $5^{\circ} \mathrm{C} / \mathrm{min}$. The inlet temperature was $230^{\circ} \mathrm{C}$. The column was eluted with $\mathrm{N}_{2}$ gas at a flow rate of $35 \mathrm{ml} / \mathrm{min}$. Toluene and cyclohexane were identified via a comparison of the retention times with the standard. Degradation experiments were performed in triplicate.

\subsection{Morphological and Biochemical Analysis}

The morphological properties of four isolated VOC strains were examined using light microscopy. The color and surface texture of the four VOC strains on the LB agar plate and the $\mathrm{BH}$ agar plate with $1 \%(\mathrm{v} / \mathrm{v})$ VOCs were investigated. An assessment of the biochemical properties was performed by mainly using the ABI 50-CHB kit (Biomerieux, France) developed by the Korean Culture Center of Microorganisms (KCCM).

\subsection{Sequencing of 16S rRNA Genes and Phylogenetic Analysis}

Genomic DNA was extracted and 16S rDNA sequences were amplified by PCR using the following primers: F27 (5'-CAG CGG TAC CAG AGT TTG ATC CTG GCT CAG-3') and R1492 (5'-CTC TCT GCA GTA CGG CTA CCT TGT TAC GAC TT-3'). The gene sequences obtained in this study were compared to nucleic acid in the GenBank database using the Blastn search tools of 
the BLAST program (www.ncbi.nlm.nih.gov/BLAST). Multiple alignments of sequences and construction of Neighbor-Joining phylogenetic trees were performed through the clustalW2 website (http://www.ebi.ac.uk /Tools/msa/clustalw2/). The molecular evolutionary distance matrices were calculated using MEGA ver.4 software [18]. The stability relationship was assessed by a bootstrap analysis of 1000 replications. The 16S rDNA sequences have been deposited in the GenBank database under the following accession numbers: JQ653962, JQ653963, JQ653964, and JQ653965. The accession numbers for reference sequences are shown in Figure 1.

\subsection{Statistical Analysis}

Statistical significance analysis of Toluene and cyclohexane degradation of four Bacillus VOC strains was assessed at the 0.05 level using a Tukey's test. This test was performed to examine the significant differences in the amount of toluene and cyclohexane between for 0 day (solid bar) vs 7 days (open bar). Stars indicated sta- tically significant differences from 0 day at $\mathrm{P}<0.05(*)$, $\mathrm{P}<0.01(* *)$.

\section{Results and Discussion}

Ninety types of strains of VOC-tolerant bacteria were isolated from the loess component of a biofilter (IL WOO TECH Co., Ltd.) that was exposed to gasoline for $50 \mathrm{~h}$ in a sealed container. The strains were isolated with differentiated morphologies on LB- or TSB-agar plates at $30^{\circ} \mathrm{C}$ and $37^{\circ} \mathrm{C}$. On the basis of an analysis of the partial sequencing of $16 \mathrm{~S}$ rDNA and a subsequent databank search, we sorted out the strains that may have the ability to degrade organic solvent. From these, strains of genera Pseudomonas, Rhodococcus, etc., were excluded from this study, because VOCs or solvent-degrading microorganisms in these genera were already well studied. Through this process, 35 strains were selected and labelled according to the series VOC01 to VOC35. Table 1 lists the 14 strains that were tolerant to $1 \%(\mathrm{v} / \mathrm{v})$ of toluene, cyclohexane, or benzene on an LB agar plate, and that

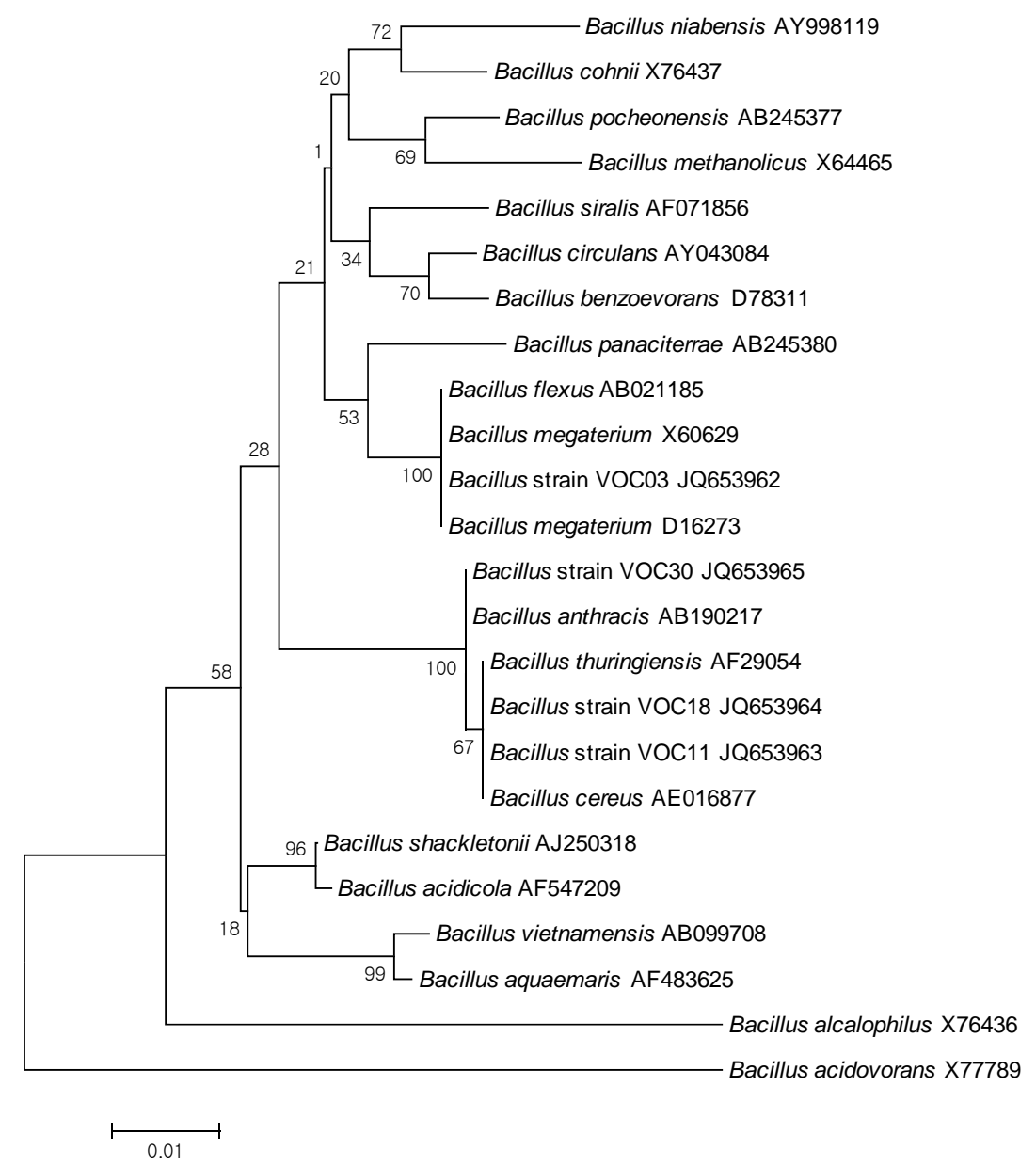

Figure 1. Phylogenetic tree of Bacillus strains VOC03, VOC11, VOC18, VOC30 and related species constructed on the basis of $16 \mathrm{~S}$ rDNA sequences using the neighbour-joining method. Bar, $0.01 \mathrm{~K}_{\text {nuc }}$ unit. Bootstrap values (1000 re-samplings) are shown at the branch points. 
Table 1. Isolation of VOCs-degrading bacteria from loess filter. VOCs (toluene, cyclohexane and benzene)-degrading or resistent bacterial strains were screened on VOCs-containing agar plate, and their 16S rRNA sequences were analyzed.

\begin{tabular}{|c|c|c|c|c|c|c|c|c|c|c|}
\hline \multirow{2}{*}{ Strain name } & \multicolumn{2}{|c|}{ Related strain ${ }^{*}$} & \multicolumn{2}{|c|}{ Toluene } & \multicolumn{2}{|c|}{ Cyclohexane } & \multicolumn{2}{|c|}{ Benzene } & \multicolumn{2}{|c|}{ Colony } \\
\hline & Related strain & Similarity (\%) & $\mathrm{BH}$ & LB & $\mathrm{BH}$ & LB & $\mathrm{BH}$ & LB & Color & Surface \\
\hline VOC03 & B. megaterium & 99 & ++ & $(-)$ & ++ & ++ & + & ++ & Beige & Rough and rigid \\
\hline VOC05 & B. megaterium & 99 & ++ & $(-)$ & ++ & +++ & - & - & Ivory & Smooth \\
\hline VOC04 & B. mycoides & 99 & + & $(-)$ & ++ & + & - & $(-)$ & Ivory & Sticky \\
\hline VOC10 & B. thuringiensis & 99 & ++ & ++ & + & +++ & + & $(-)$ & Ivory & Smooth \\
\hline VOC11 & Bacillus sp. & 98 & + & + & ++ & +++ & + & + & Ivory & Sticky \\
\hline VOC18 & Bacillus sp. & 99 & ++ & + & ++ & +++ & + & +++ & Orange yellow & Wrinkled \\
\hline VOC20 & Bacillus sp. & 99 & + & ++ & ++ & ++ & + & + & white & Sticky \\
\hline VOC21 & B. thuringiensis & 99 & - & + & ++ & + & - & +++ & Ivory & Smooth \\
\hline VOC22 & B. thuringiensis & 98 & + & $(-)$ & $+[++]$ & + & + & ++ & White & Smooth \\
\hline $\mathrm{VOC} 23$ & B. cereus & 99 & + & $(-)$ & ++ & + & - & +++ & Ivory & Smooth \\
\hline VOC24 & B. simplex & 99 & $+[++]$ & ++ & $+[++]$ & +++ & + & $(-)$ & Ivory & Rough \\
\hline VOC26 & B. cereus & 99 & - & ++ & ++ & ++ & - & $(-)$ & Ivory & Sticky \\
\hline VOC28 & Bacillus sp. & 99 & ++ & ++ & + & +++ & + & $(-)$ & Ivory & Rough \\
\hline VOC30 & Bacillus sp. & 99 & ++ & $(-)$ & ++ & +++ & + & ++ & Ivory & Smooth \\
\hline
\end{tabular}

could simultaneously grow on the $1 \%(\mathrm{v} / \mathrm{v})$ of the same VOC as the sole $\mathrm{C}$ source on the $\mathrm{BH}$ minimal agar plate. All these strains belonged to the genus Bacillus. Some other strains of genera Staphylococcus, Micrococcus, and Anthrobacter were tolerant to VOCs on the LB plate, but they could not grow on VOCs on the $\mathrm{BH}$ minimal agar plate (not shown). The 14 Bacillus strains exhibited a differentiated morphology in the color and form of the colony: white, ivory, pale pink, beige, or yellow in colony color; rough, smooth, rigid, soft, sticky, moisture-less or wrinkled on the colony surface (Table 1). The growth of strains on the VOCs on minimal agar (Table 1) provided a clue that the strains could use cyclohexane, toluene, or benzene as a carbon source. The degradation of toluene and cyclohexane by the VOC strains was directly determined by GC, and the culture was grown in sealed bottles (Figure 2).

For this measurement, we selected four strainsVOC03, VOC11, VOC18, and VOC30 - that grew dominant, overall, on toluene, cyclohexane, and benzene, simultaneously, and that were morphologically obviously different from each other (Table 2).

Toluene and cyclohexane were degraded similarly. The VOC18 strain degraded toluene best, which decreased by $29.6 \%$ (59.8 ppm of the total $202.3 \mathrm{ppm}$ ) after seven days of incubation (Figure 2). The VOC30 and VOC11 strains degraded $21.1 \%$ and $11.6 \%$ of the toluene, respectively. In the case of cyclohexane, the VOC 18 and
VOC11 strains degraded similarly, with a $25.9 \%(54.8$ ppm of the total $211.3 \mathrm{ppm}$ ) and a $25.6 \%$ (57.6 ppm of the total $225.5 \mathrm{ppm}$ ) reduction of cyclohexane, respectively; the VOC30 strain resulted in a $21.2 \%$ reduction of cyclohexane (44.9 ppm of the total $211.6 \mathrm{ppm}$ ). The degradation of toluene by the VOC18 strain was significantly different as compared to the condition on the 0 day $(p<0.01)$. However, the degradation of both cyclohexane and toluene by the VOC03 strain was not significant $(\mathrm{p}<0.05)$. The incubation under airtight conditions was inevitable for the degradation experiment, but the condition had a limited oxygen supply. The degradation of cyclohexane and toluene was sensitive to the lack of oxygen. For aerobic cleavage, aromatic rings are attacked with the help of molecular O2 by oxygenases [19]. The three VOC-degrading strains (VOC11, VOC18, and VOC30) shown in Figure 2 were able to grow on toluene, cyclohexane, benzene, or gasoline as a sole carbon source in a BH minimal liquid medium with vigorous aeration (Table 3). The strains generally grew well with cyclohexane, but less well with benzene. Cyclohexane may be relatively less toxic than the other tested VOCs be-cause the $\log P_{\text {ow }}$ value of cyclohexane (3.30) is higher than that of toluene (2.69) and benzene (2.13). The solvent with $\log P_{\text {ow }}$ values below 4 is considered to be extremely toxic, whereas the solvent 2.13). The solvent with $\log P_{\text {ow }}$ values below 4 is considered to be extremely toxic, whereas the solvent with higher $\log P$ ow values is 


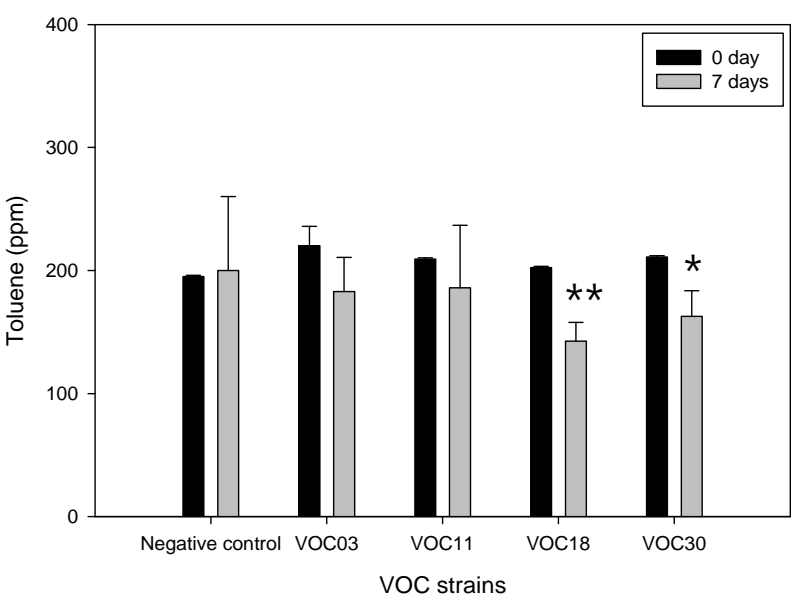

(a)

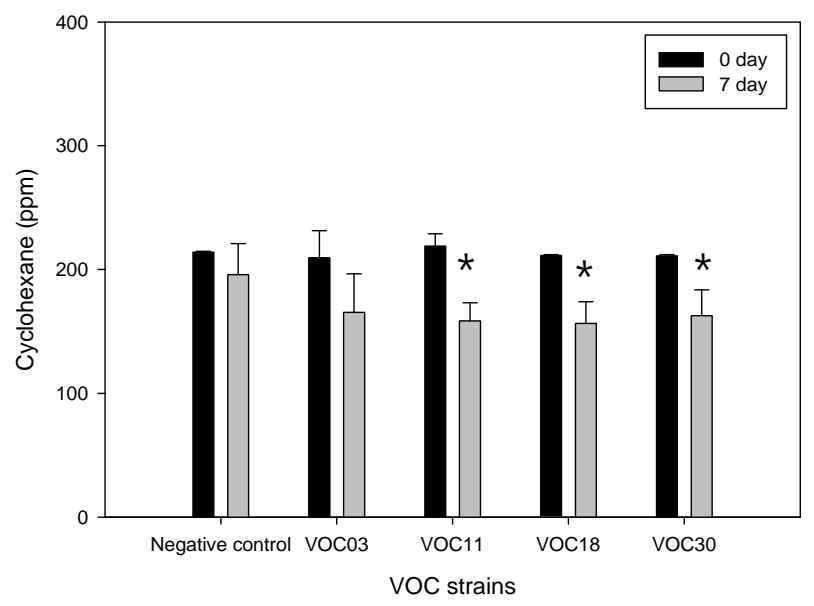

(b)

Figure 2. Toluene and cyclohexane degradation of four Bacillus VOC strains. The degradation of $200 \mathrm{ppm}$ of each toluene (a) and cyclohexane (b) by strain VOC03, VOC11, VOC18, were observed in BH medium in air-tight bottle during incubation at $30^{\circ} \mathrm{C}$ with shaking. The amount of toluene and cyclohexane was analyzed by GC for 0 day (solid bar) vs 7 days (open bar). N/C is the negative control without strains. Stars indicated statically significant differences from 0 day at $\mathbf{P}<0.05(*), P<$ $0.01(* *)$.

Table 2. Colony morphology of VOC03, VOC11, VOC18, and VOC30 growth on LB.

\begin{tabular}{cccccc}
\hline Media & & VOC03 & VOC11 & VOC18 & VOC30 \\
\hline & Colour & Beige & Ivory & Orange-yellow & Ivory \\
LB & Surface texture & Rough and rigid & Sticky & Sticky and wrinkled & Smooth \\
& Photo & & & & \\
\hline
\end{tabular}

Table 3. The growth of bacillus stains on VOC. The strains were cultivated in BH liquid medium with $1 \%$ of toluene, cyclohexane, benzene or gasoline at $30^{\circ} \mathrm{C}$ for 4 day on a shaking incubator $(180 \mathrm{rpm})$.

\begin{tabular}{|c|c|c|c|c|c|c|}
\hline & \multicolumn{2}{|c|}{ VOC11 } & \multicolumn{2}{|c|}{ VOC18 } & \multicolumn{2}{|c|}{ VOC30 } \\
\hline & $\Delta \mathrm{OD}_{600}$ & $\mathrm{Y}_{\mathrm{sub}}[\mathrm{g} \mathrm{dw} / \mathrm{mol}]$ & $\Delta \mathrm{OD}_{600}$ & $\mathrm{Y}_{\mathrm{sub}}[\mathrm{g} \mathrm{dw} / \mathrm{mol}]$ & $\Delta \mathrm{OD}_{600}$ & $\mathrm{Y}_{\mathrm{sub}}[\mathrm{g} \mathrm{dw} / \mathrm{mol}]$ \\
\hline Without VOC & 0.01 & nd & -0.07 & nd & -0.02 & nd \\
\hline Toluene & 1.06 & 16.82 & 1.16 & 18.54 & 1.16 & 18.56 \\
\hline Cyclohexane & 1.10 & 17.82 & 1.32 & 21.41 & 1.21 & 19.64 \\
\hline Benzene & 1.05 & 14.04 & 1.30 & 17.41 & 1.01 & 13.47 \\
\hline Gasoline & 0.07 & nd & 0.18 & nd & 1.45 & nd \\
\hline
\end{tabular}

Nd: not determined; Cell dry weight $300 \mathrm{mg} / \mathrm{L}$; Y sub: Yield of subculture (g dryweight/1molecule of Carbone source).

considered less toxic [20,21]. The substrate molar yield $\left(\mathrm{Y}_{\text {sub }}\right)$ with cyclohexane was also the highest in each strain. This corresponds with the fact that the degree of reduction of cyclohexane (6.0) is higher than that of toluene (5.1) or benzene (5.0). In complete oxidation of the substrate, the higher the number of electrons per carbon atom, the more energy could be produced for growth [22]. The cell growth on gasoline was low and slow; VOC11 and VOC18 did not grow by the fourth day. It was thought that because gasoline contains a mixture of more toxic substances, it inhibited the growth more severely than cyclohexane, toluene, or benzene. In addition, the strains could also grow very well on hexane, octane, and dodecane (data not shown). Each strain has different capabilities of using each VOC.

The strains of genus Bacillus growing on VOCs were identified according to Bergey's manual of systematic bacteriology and by considering the physiological and 
biochemical characteristics. The strains were characterized by differentiated utilization patterns of sugar, sugar alcohol, acids, and sugar derivatives mainly using API 50 CHB (Table 4). Among the monosaccharides, all of the four tested strains utilized glucose, fructose, and ribose. In addition to those three common sugars, strain VOC03 can use L-arabinose, D-xylose, galactose, and D-raffinose, and strain VOC18 can use D-mannose. VOC11, VOC18, and VOC30 cannot metabolize any of the tested sugar alcohols. On the other hand, VOC03 can use glycerol, mannitol, and inositol. All four strains can use two disaccharides, namely, maltose and trehalose. In addition, strain VOC18 degrades cellobiose and sucrose, and VOC30 degrades sucrose.

Table 4. The biochemical characteristics of the 4 VOC-growing strains.

\begin{tabular}{|c|c|c|c|c|c|}
\hline Growth on & & VOC03 & VOC11 & VOC18 & VOC30 \\
\hline \multirow[t]{4}{*}{ Acids } & Citrate & - & - & - & - \\
\hline & Gluconate & - & - & - & - \\
\hline & 2-ceto-gluconate & - & - & - & - \\
\hline & 5-ceto-gluconate & - & - & - & - \\
\hline \multirow[t]{15}{*}{ Sugar } & D-Arabinose & - & - & - & - \\
\hline & L-Arabinose & + & - & - & - \\
\hline & Ribose & + & + & + & + \\
\hline & D-Xylose & + & - & - & - \\
\hline & L-Xylose & - & - & - & - \\
\hline & Galactose & + & - & - & - \\
\hline & D-Glucose & + & + & + & + \\
\hline & D-Fructose & + & + & + & + \\
\hline & D-Mannose & - & - & + & - \\
\hline & L-sorbose & - & - & - & - \\
\hline & Rhamnose & - & - & - & - \\
\hline & D-Lyxose & - & - & - & - \\
\hline & D-Tagatose & - & - & - & - \\
\hline & D-Fucose & - & - & - & - \\
\hline & L-Fucose & - & - & - & - \\
\hline \multirow[t]{10}{*}{ Sugar alcohol } & Glycerol & + & - & - & - \\
\hline & Ertythritol & - & - & - & - \\
\hline & Dulcitol & - & - & - & - \\
\hline & Inositol & + & - & - & - \\
\hline & Mannitol & + & - & - & - \\
\hline & Sorbitol & - & - & - & - \\
\hline & Adonitol & - & - & - & - \\
\hline & Xylitol & - & - & - & - \\
\hline & D-Arabitol & - & - & - & - \\
\hline & L-Arabitol & - & - & - & - \\
\hline \multirow[t]{6}{*}{ Disaccharide } & Cellobiose & + & - & + & - \\
\hline & Melibiose & + & - & - & - \\
\hline & Saccharose & + & - & + & + \\
\hline & Trehalose & + & + & + & + \\
\hline & $\beta$-Gentiobiose & + & - & - & - \\
\hline & D-Turanose & + & - & - & - \\
\hline Trisaccharide & Melezitose & - & - & - & - \\
\hline Polysaccharide & Glycogen & + & + & + & + \\
\hline Amino sugar & N-Acetyl-glucosamine & + & + & + & + \\
\hline \multirow[t]{7}{*}{ Glucoside } & $\beta$-Methyl-xyloside & - & - & - & - \\
\hline & $\alpha$-Methyl-D-mannoside & - & - & - & - \\
\hline & $\alpha$-Methyl-D-glucoside & - & - & - & - \\
\hline & Amygdaline & + & - & + & - \\
\hline & Arbutine & + & + & + & + \\
\hline & Esculine & + & + & + & + \\
\hline & Salicine & + & + & + & + \\
\hline \multirow[t]{2}{*}{ Synthetic opioid } & Amidon & + & + & + & + \\
\hline & Insuline & - & - & - & - \\
\hline $\begin{array}{l}\text { Similarity to suggested } \\
\text { species }\end{array}$ & & B. megaterium $99.4 \%$ & $\begin{array}{l}\text { B. mycoides } 88.5 \% \text {; } \\
\text { B. cereus } 7.4 \%\end{array}$ & B. cereus $99.3 \%$ & $\begin{array}{l}\text { B. mycoides } 71.9 \% \text {; } \\
\text { B. cereus } 12.8 \% \text {; } \\
\text { B. antracis } 12.5 \%\end{array}$ \\
\hline
\end{tabular}


Strain VOC03 can use all of the tested disaccharides. With other polysaccharides, sugar acids, glucosides, and sugar derivatives, all four Bacillus strains exhibited a similar utilization pattern except for amygdaline. According to this carbohydrate-utilization pattern, strain VOC03 was related with B. megaterium with $99.4 \%$ identity, and VOC18, to B. cereus with $99.3 \%$ identity. The result of VOC11 revealed an $88.5 \%$ identity to $B$. mycoides and only a $7.4 \%$ identity to $\mathrm{B}$. cerues, but additional tests would be required to determine whether it was B. thuringiensis. Similarly, VOC30 has a $71.9 \%$ identity to $B$. mycoides but only a $12.8 \%$ and $12.5 \%$ identity to B. cereus and B. anthracis, respectively. Again, an additional test was needed to identify $B$. thuringiensis, $B$. cereus, or B. mycoids.

The strains were further identified by classification using 16S rDNA/sequence analysis; phylogenetic relationships were derived from 16S rDNA sequence analysis of strains VOC03, VOC11, VOC18, and VOC 30 with respect to species within the genus Bacillus (Figure 1). The phylogenetic tree was constructed using the neighborjoining method [23]. 16S rRNA gene sequences of strain VOC03 were most closely related to $B$. megaterium, and the sequence similarity was $99.5 \%$ with $B$. megaterium strain IAM 13418. It was consistent with the identification of physiological characteristics (Table 4). Strain VOC03 utilized different carbon sources such as L-arabinose, ribose, D-xylose, galactose, D-glucose, Dfructose, inositol, and mannitol, which was consistent with Tay et al.'s report of an identified PHA accumulating bacteria strain having $99 \%$ homology of B. megaterium [24]. Both VOC11 and VOC18 were evolutionarily related to B. thuringiensis and B. cereus. Among them, VOC18 was shown to be physiologically identical to B. cereus (Table 4) while VOC11 was shown to have slight physiological differences from $B$. cereus. The sequence similarity was $99.7 \%$ with $B$. thuringiensis strain IAM 12077, and therefore, VOC11 is likely to be a strain of $B$. thuringiensis. This supported the idea that bacterial and fungal isolates from a PAH-degrading bacterial consortium have been reported to grow on or mineralize volatile organic compounds $[25,26]$. The $16 \mathrm{~S}$ rDNA sequence of the VOC30 strain was phylogenetically related to $B$. anthracis, B. thuringiensis, or B. cereus (Figure 1), but its physiological characteristics showed there to be a low possibility for it to be a B. cereus (12.8\%) or B. anthracis $(12.5 \%)$ (Table 4). In addition, the BLAST result of VOC30 showed it to be highly similar (99.4\%) to the $B$. thuringiensis strain IAM 12077.

\section{Conclusion}

We used a filter to isolate four Bacillus strains from loess - VOC03, VOC11, VOC18, and VOC30. The VOC11,
VOC18, and VOC30 strains degraded both cyclohexane and toluene, and they were able to use the VOCs as a $\mathrm{C}$-source. The VOC18 strain grew on cyclohexane or toluene best, and it degraded the VOCs faster than the other strains. The physiological characteristics and phylogenetic analysis showed that the VOC18 strain was a strain of B. cereus, and both VOC11 and VOC30 were strains of $B$. thuringiensis. The VOC03 strain, which was determined as a strain of B. megaterium, was not able to use cyclohexane and toluene, although it was highly tolerant to the VOCs. Soil bacteria such as B. cereus VOC18, B. thuringiensis VOC11, and B. thuringiensis VOC30 have been continuously eliminating aromatic VOCs such as cyclohexane or toluene in our soil environment. This demonstrates the need to conserve ecologically soil area, especially in industrial cities. In addition, the efficient VOC-degrading strain VOC18 from loess may have the industrial applicable possibility in the bioremediation of VOC pollutants.

\section{Acknowledgements}

This work was supported by the work was supported by the Ewha Global Top 5 Grant 2012 of Ewha Womans University (grant 2012-1783-11). We are grateful to Prof. Kyung Suk Cho (Ewha W. University) for support of GC, to Sung Il Kim (IL WOO TECH Co., Ltd.) for supply of loess filter, and to Mi Na Rhee and Hee Jong Lee for experimental support during undergraduate internship.

\section{REFERENCES}

[1] R. G. Derwent, "Sourses, Distributions, and Fates of VOCs in Atmosphere," Environmental Science Technology, Vol. 4, 1995, pp. 1-15.

[2] P. Kuran and L. Sojak, "Environmentel Analysis of Volatile Organic Compounds in Water and Sediment by Gas Chromatography," Journal of Chromatograph A, Vol. 733, 1996, pp. 119-141.

[3] J. Dewulf and H. Van Langenhove, "Analytical Techniques for the Determination and Measurement Data of 7 Chlorinated C-1- and C-2-Hydrocarbons and 6 Monocyclic Aromatic Hydrocarbons in Remote Air Masses: An Overview," Atmospheric Envirnment, Vol. 31, 1997, pp. 3291-3307.

[4] L. K. Sahu, "Volatile Organic Compounds and Their Measurements in the Troposphere," Current Science, Vol. 102, No. 12, 2012, pp. 1645-1649.

[5] J. F. Muller, "Geographical-Distribution and SeasonalVariation of Surface Emissions and Deposition Velocities of Atmospheric Trace Gases," Journal of Geophysical Research, Vol. 97, 1992, pp. 3787-3804.

[6] S. D. Picco, J. J. Watson and J. W. Jones, "A Global Inventory of Volatile Organic-Compound Emissions from Anthropogenic Sources," Journal of Geophysical Research, Vol. 97, 1992, pp. 9897-9912. 
[7] M. Braeckevelt, E. M. Seeger, H. Paschke, P. Kuschk and M. Kaestner, "Adaptation of a Constructed Wetland to Simultaneous Treatment of Monochlorobenzene and Perchloroethene," International Journal of Phytoremediation, Vol. 13, No. 10, 2011, pp. 998-1013. doi:10.1080/15226514.2010.549860

[8] E. H. Lee, J. Kim, K. S. Cho, Y. G. Ahn and G. S. Hwang, "Degradation of Hexane and Other Recalcitrant Hydrcarbons by a Novel Isolate, Rhodococcus sp. EH831," Environmental Science and Polluting Research, Vol. 17, No. 1, 2010, pp. 64-77. doi:10.1007/s11356-009-0238-x

[9] H. de Klerk and A. C. van der Linden, "Bacterial Degradation of Cyclohexane Participation of a Co-Oxidation Reaction," Antonie van Leeuwenhoek, Vol. 40, No. 1, 1974, pp. 7-15. doi:10.1007/BF00394548

[10] M. S. Anderson, R. A. Hall and M. Griffin, "Microbial Metabolism of Alicyclic Hydrocarbons: Cyclohexane Catabolism by a Pure Strain of Pseudomonas sp.," Journal of General Microbiology, Vol. 120, 1980, pp. 89-94.

[11] M. K. Trower, R. M. Buckland, R. Higgins and M. Griffin, "Isolation and Characterization of a Cyclohexane-Metabolizing Xanthobacter sp.," Applied Environmental Microbiology, Vol. 49, 1985, pp. 1282-1289.

[12] P. E. Rouviere and M. W. Chen, "Isolation of Brachymonas Petroleovorans CHX, a Novel Cyclohexane-Degrading Beta-Proteobacterium," FEMS Microbiological Letters, Vol. 227, 2003, pp. 101-106.

[13] M. Matsumoto, J. A. de Bont and S. Isken, "Isolation and Characterization of the Solvent-Tolerant Bacillus Cereus Strain R1," Journal of Bioscience and Bioengineering, Vol. 94, 2002, pp. 45-51.

[14] M. L. Paje, B. A. Neilan and I. Couperwhite, "A Rhodococcus Species That Thrives on Medium Saturated with Liquid Benzene," Microbiology, Vol. 143, No. 9, 1997, pp. 2975-2981.

[15] E. H. Lee and K. S. Cho, "Characterization of Cyclohexane and Hexane Degradation by Rhodococcus sp. EC1," Chemosphere, Vol. 71, 2008, pp. 1738-1744.

[16] C. Singh and J. Lin, "Isolation and Characterization of Diesel Oil Degrading Indigenous Microorganisms in Kwazulu-Natal, South Africa," African Journal of Biotechnology, Vol. 7, No. 12, 2008, pp. 1927-1932.
[17] L. D. Bushnell and H. F. Haas, "The Utilization of Certain Hydrocarbons by Microorganisms," Journal of Bacteriology, Vol. 41, 1941, pp. 653-673.

[18] K. Tamura, J. Dudley, M. Nei and S. Kumar, "MEGA4: Molecular Evolutionary Genetics Analysis (MEGA) Software Version 4.0," Molecular Biological Evolution, Vol. 24, 2007, pp. 1596-1599.

[19] G. Fuchs, M. Boll and J. Heider, "Microbial Degradation of Aromatic Compounds-From One Strategy to Four," Natural Review of Microbiology, Vol. 9, 2001, pp. 803816.

[20] J. L. Ramos, E. Duque, M. T. Gallegos, P. Godoy, M. I. Ramos-González, A. Rojas, W. Terán and A. Segura, "Mechanisms of Solvent Tolerance in Gram-Negative Bacteria," Annual Reviews in Microbiology, Vol. 56, 2002, pp. 743-768.

[21] H. V. Westehoff, K. J. Hellingwerf and K. van Dam, "Thermodynamic Efficiency of Microbial Growth Is Low but Optimal for Maximal Growth Rate," Proceedings of $\mathrm{Na}$ tional Academy of Science of USA, Vol. 80, 1983, pp. 305-309.

[22] M. M. Lazaroaie, "Mechanisms Involved in Organic Solvent Resistance in Gram-Negative Bacteria," World Academic Science, Engineering Technology, Vol. 54, 2009, pp. 648-658

[23] J. Felsenstein, "Phylogeny Inference Package (PHYLIP). Version 3.5," University of Washington, Seattle, 1993.

[24] B. Y. Tay, B. E. Lokesh, C. Y. Lee and K. Sudesh, "Polyhydroxyalkanoate (PHA) Accumulating Bacteria from the Gut of Higher Termite Macrotermes carbonarius (Blattodea: Termitidae),"World Journal of Microbiol Biotechnology, Vol. 26, 2010, pp. 1015-1024.

[25] A. Janbandhu and M. H. Fulekar, "Biodegradation of Phenanthrene Using Adapted Microbial Consortium Isolated from Petrochemical Contaminated Environment," Journal of Hazard Materials, Vol. 187, 2011, pp. 333-340.

[26] Y. Lin and L. X. Cai, "PAH-degrading Microbial Consortium and Its Pyrene-Degrading Plasmids from Mangrove Sediment Samples in Huian, China," Marine Polluting Bullutin, Vol. 57, 2008, pp. 703-706. 